\title{
INFLUENCE OF FLUORIDE MOUTHWASH CONTAINING NANOHYDROXYAPATITE ON THE DENTIN INTERFACE OF DIRECT RESTORATIONS: A RANDOMIZED CONTROLLED CROSSOVER IN SITUSTUDY
}

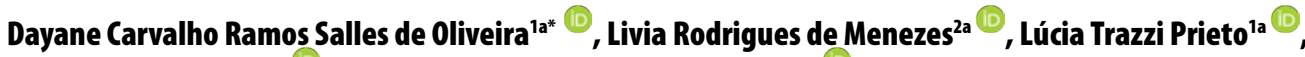

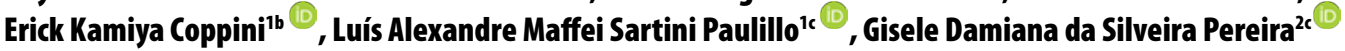 \\ 'Department of Restorative Dentistry, Piracicaba Dental School, State University of Campinas, Piracicaba, SP, Brazil \\ ${ }^{2}$ Federal University of Rio de Janeiro, Rio de Janeiro, RJ, Brazil \\ aPhD, Assistant Professor \\ bPhD \\ PhD, Associate Professor
}

ABSTRACT

DOI: https://doi.org/10.25241/stomaeduj.2019.6(1).art.3

Introduction: To evaluate the influence of a fluoride mouthwash containing nanohydroxyapatite on the dentin bonding interface microhardness of direct resin composite restorations in situ.

Methodology: Forty human premolars were sectioned and restored with a three-step adhesive system or a self-etch adhesive system and a conventional resin-based composite. After the initial microhardness measurements on the samples' dentin interface, the restored samples were fixed in a crylic palate a ppliances for a $\mathrm{n}$ in situ experiment.

Ten volunteers participated in a randomized double-blinded crossover study using the palate appliances and a placebo fluoride mouthwash for one week, one-week wash-out, and one-week fluoride mouthwash containing nanohydroxyapatite. The final microhardness measurements were evaluated, and data were analyzed by ANOVA and Tukey's test submitted for multiple comparisons ( $\alpha=0.05 ; 0.8$ power).

Results: There were statistically significant differences be tween the mouthwash treatments with both adhesive systems tested $(p<0.001)$. The nanohydroxyapatite used in the mouthwash was an influencing actor on the dentin interface microhardness of resin composite restorations.

Conclusion: The fluoride m outhwash containing the $n$ anohydroxyapatite $w$ as a ble to increase the dentin microhardness of the direct resin composite restorations regardless of the adhesive system used.

Keywords: Dental materials; composite resins; dental adhesive; mouthwashes.

OPEN ACCESS This is an Open
Access article under the CC BY-NC
4.0 license.
Peer-Reviewed Article
Citation: de Oliveira DCRS, de Menezes LR,
Prieto LI, Coppini EK, Sartini Paulillo LAM,
da Silveira Pereira GD. Influence of fluoride
mouthwash containing nanohydroxyapatite
on the dentin interface of direct restorations:
a randomized controlled crossover in situ
study. Stoma Edu J. 2019;6(1):24-28
Received: February 09, 2019
Revised: February 28, 2019
Accepted: March 18, 2019
Published: March 19, 2019
*Corresponding author:
Dayane C.R.S. de Oliveira
Department of Restorative Dentistry,
Piracicaba Dental School
State University of Campinas, 901 , Limeira
Ave.SP-13414-903 Piracicaba, Brazil
Tel/ Fax: +55 (019) 2106-5341
e-mail: dayoli87@ @gmail.com
Copyright: $\odot 2019$ the Editorial Council
for the Stomatology Edu Journal.

\section{Introduction}

Dental enamel etching increases the surface energy, which facilitates the penetration of the adhesive system and the adhesion to this substrate[1]. Adhesion to the enamel substrate is more efficient than to the dentin substrate since it is composed of an inorganic matrix, while dentin presents a more significant amount of organic matrix composed mainly of collagen and water [2]. Moisture control, maintenance of the permeable collagen to the adhesive system penetration, and demineralization of acid etching are essential factors to improve the transition zone, called the hybrid layer [3], which is the primary mechanism of adhesion to the dentin substrate [4]. Several factors can influence the hybrid layer development. When moisture control is performed incorrectly, the penetration of the monomers into the demineralized dentin is incomplete, resulting in failure areas. This increases the risk of nanoleakage through the pores present in the adhesive interface of this defective hybrid layer. It can cause postoperative sensitivity and reduce the longevity of the direct restorations $[5,6]$. The presence of failure areas on the adhesive interface enables the infiltration of various harmful agents, as well as oral fluids, to infiltrate these gaps. Therefore, ions, such as calcium and fluoride, present in saliva or solutions used as mouthwashes [4] can also penetrate and fill the micro porosity present in the hybrid layer, thus decreasing its permeability [8-10]. It becomes evident that evaluating whether or not the use of mineralizing agents allows adducing within the hybrid layer of ions that can fill the nanopores present in the dentin interface is essential. Thus, this study assessed the influence of a fluoride mouthwash containing nanohydroxyapatite on the dentin bonding interface microhardness of direct resin composite restorations. The null hypothesis was that the use of the fluoride mouthwash containing nanohydroxyapatite will not influence the dentin interface microhardness of direct resin composite resin restorations.

\section{Methodology}

\subsection{Ethical aspects}

This in situ, double-blinded, single center, randomized controlled crossover study was authorized by the 


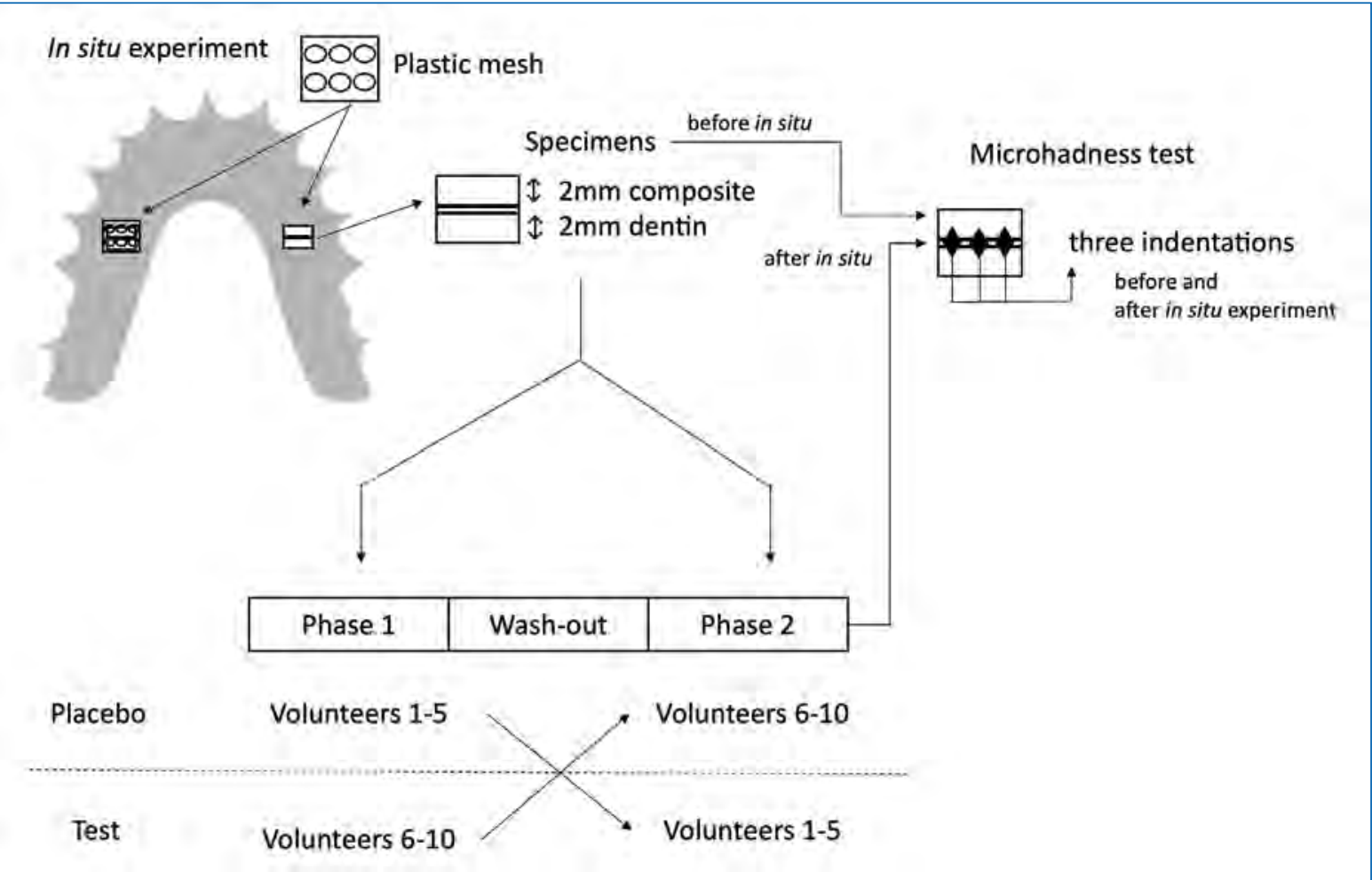

I Figure 1. Flow diagram with a systematic illustration of the crossover in situ model and experimental design.

local Institutional Ethics Committee of the Piracicaba Dental School, Piracicaba, SP, Brazil (protocol \#149/2012). A written informed consent was obtained from all volunteers in the study. Volunteers had the right to withdraw from the study at any time and for any reason without prejudice.

\subsection{Preparation of the specimens}

Forty human premolars recently extracted because of orthodontic reasons were donated to this study. The teeth were stored for $24 \mathrm{~h}$ in a $0.1 \%$ thymol solution at $37^{\circ} \mathrm{C}$, then stored in distilled water until the beginning of the study. The coronal portion was separated from the root through a straight section on the long axis of the tooth using a metallographic precision cutter (Isomet 1000, Buehler Ltd., Buff Lake, IL, USA) with a diamond saw under constant irrigation. To obtain a flat dentin surface, the occlusal surfaces were ground with sandpaper silicon carbide ( $\mathrm{SiC}$ \#320 and \#400 in a metallographic polisher sander cooled with water. Then, $4 \times 4 \mathrm{~mm}$ dentin blocks were obtained with $2 \mathrm{~mm}$ of thickness. After an ultrasound bath to remove the sander debris, the standardization of the smear layer was performed on the dentin flat by manually rubbing with SiC wet sandpaper \#1000 and \#1200 for 15 s per sandpaper grit. The dentin blocks were kept in $100 \%$ relative humidity at $37^{\circ} \mathrm{C}$. This study used the total etching technique with a three-step adhesive system (Scotchbond Multi-Purpose Plus, 3M ESPE, St. Paul, MN, USA) and a self-etch adhesive system (Clearfill SE Bond, Kuraray, Tokyo, Japan) with a nanofilled resin composite (Filtek Z350 XT, 3M ESPE, St. Paul, MN, USA). All clinical and laboratory steps were performed at the Restorative Department of Piracicaba Dental School,
UNICAMP, Piracicaba, SP, Brazil. For the three-step adhesive system, the excess water was removed by capillary action with an absorbent paper. The dentin etching was performed with $35 \%$ phosphoric acid for $15 \mathrm{~s}$, followed by rinsing with abundant water for $30 \mathrm{~s}$. The moisture was controlled with a moist cotton ball. Both adhesive systems were used according to the manufacturers' instructions with a microbrush and light cured for $20 \mathrm{~s}$ with a LED light source (Radii, SDI, Victoria, Australia). The restoration was carried out with a single $2 \mathrm{~mm}$ increment of the resin composite in a $4 \times 4 \mathrm{~mm}$ acetate pattern to standardize the direct restorations, and then light-cured for $20 \mathrm{~s}$. All samples were stored in $100 \%$ relative humidity at $37^{\circ} \mathrm{C}$ for $24 \mathrm{~h}$.

\subsection{In situ study}

This in situ, double-blinded, single center, randomized controlled crossover study was carried out for three weeks, including a one-week fluoride nanohydroxyapatite-free phase, a one-week washout period and a one-week fluoride nanohydroxyapatiteactive phase. A total of 10 adult volunteers (mean age $23.3 \pm 2.3$ yrs; 5 females and 5 males) were recruited in the Piracicaba Dental School, UNICAMP, Piracicaba, SP, Brazil and in the Dental School of the Federal University of Rio de Janeiro, Rio de Janeiro, RJ, Brazil. All volunteers had confirmed satisfactory oral health, did not use any medication, did not have any systemic disease or pregnancy, and did not use any mouthwash or toothpaste except as provided for the research. In this study, all volunteers completed the study, no participants were excluded or withdrawn from the study. Impressions of all the volunteers' mouths were made to obtain a model to make a palate acrylic 
I Table 1. Means (standard deviations) values for microhadness test (KHN).

\begin{tabular}{lcc}
\hline \multicolumn{1}{c}{ Experimental Group } & \multicolumn{2}{c}{ Microhardness } \\
\cline { 2 - 3 } & \multicolumn{1}{c}{ Initial } & Final \\
\hline Clearfill SE (+ testing mouthwash) & $42.59(0.5) \mathrm{Ba}$ & $44.33(0.6) \mathrm{Aa}$ \\
Clearfill SE (+ placebo mouthwash) & $41.29(0.5) \mathrm{Aa}$ & $40.4(1.0) \mathrm{Ab}$ \\
ScotchBond MP (+ testing mouthwash) & $42.15(0.4) \mathrm{Bb}$ & $45.42(0.6) \mathrm{Aa}$ \\
ScotchBond MP (+ placebo mouthwash) & $39.66(0.6) \mathrm{Aab}$ & $40.35(0.5) \mathrm{Ab}$ \\
\hline
\end{tabular}

${ }^{*}$ Means followed by different capital letters in the same line and small letters in the same column were significantly different $(p<0.05)$.

appliance for the upper arch. In each plate two cavity sites were fabricated where the specimens were fixed. All sites were positioned posterior to the incisive papillae to avoid contact between the tongue and the specimens. Every site had a $4.5 \times 4.5 \mathrm{~mm}$ uniform gap with $4 \mathrm{~mm}$ in depth covered by a plastic mesh to allow free contact of the saliva with the specimens, but protection from mechanical disturbance. New specimens were inserted into the appliance before phase 2 . The specimens were randomly assorted and allocated into two groups: experimental or placebo. A systematic illustration of the crossover in situ model and the experimental design can be observed in Fig. 1. In the first week half of the volunteers were randomly allocated to use the fluoride mouthwash containing nanohydroxyapatite, while the other half to use the placebo fluoride mouthwash containing no nanohydroxyapatite. After the second week "wash-out" to neutralize the fluoride agent and the microhardness readings, the groups were reversed and conducted as described in the first week. The volunteers who used the placebo fluoride mouthwash in the first week used the tested mouthwash in the second week and vice versa. The study products and tested materials were provided as coded packages labeled with participants number and study period. None of the volunteers, in situ study instructors or in situ study monitors that were participating in the study knew the type of group (placebo or test) being tested until all procedures were finalized. The in situ study coordinators were responsible for blinding all participants and labeling the study products and tested materials. The mouthwashes used in the study were prepared by FGM Produtos Odontológicos (89219-501 Joinville, SC, Brasil). The placebo fluoride mouthwashes were produced with the same color, viscosity and taste compared to the tested mouthwash. The placebo mouthwash and the tested one contained the same components, including the fluoride and the potassium nitrate, but only the tested mouthwash contained the nanohydroxyapatite, as in the Desensibilize Nano $P$ (FGM Produtos Odontológicos, 89219-501 Joinville, SC, Brasil). The volunteers were instructed to swish $20 \mathrm{~mL}$ of the mouthwash around their mouth for thirty seconds then spit it out with no rinsing. The volunteers were also instructed to use it twice a day, every day, after brushing and flossing their teeth in the morning and at night. All volunteers were instructed to brush their teeth using the same toothpaste containing no fluoride during all study. The volunteers should wear the appliance for more than $20 \mathrm{~h}$ per day, removing it only while eating or brushing. The volunteers were instructed to rinse off their appliance and store it in artificial saliva while not in use.

\subsection{Microhardness readings (KHN)}

The Knoop Microhardness of the dentin interface (illustrated in Fig. 1) was examined using a microhardness tester (HMV-2, Shimadzu, Kyoto, Japan) with a $50 \mathrm{~g}$ load for $15 \mathrm{~s}$ in three equally spaced points on each sample. A blinded trained operator performed all the microhardness measurements before and after the crossover in situ experiment. The microhardness of each sample was taken from the arithmetic mean of the three readings.

\subsection{Statistical analyses}

The sample size was calculated according to a pilot test to provide a power of 0.8. The power was calculated according to power analysis. The data were subjected to ANOVA and Tukey's test with a limit of $5 \%$ probability of making decisions.

\section{Results}

ANOVA showed a significant difference between the tested groups (placebo/tested mouthwash $x$ time) $(p<0.001)$. Mean values taken from Tukey's test are shown in Table 1. As could be observed, the nanohydroxyapatite used in the mouthwash was an influencing factor on the dentin interface microhardness of resin composite restorations. The fluoride mouthwash containing the nanohydroxyapatite managed to increase the dentin interface microhardness of direct resin composite restorations regardless of the adhesive system used, while the placebo fluoride mouthwash solution containing no nanohydroxyapatite had no influence on dentin interface microhardness.

\section{Discussion}

The present study clinically investigated the influence of a fluoride mouthwash containing nanohydroxyapatite on the dentin bonding interface microhardness of direct resin composite restorations in situ. There are several in vitro studies showing 
possible effects of mouthwashes in composite restorations overtime [11-14]. However, there is no consensus about this topic, and the reason for this is that different mouthwashes contain different components. The crossover in situ model allowed to access the real effects in the oral cavity. Moreover, the use of the same fluoride mouthwash composition varying only theaddition ornot of nanohydroxyapatite, allowed to access the real influence of the different mineralizing agents on the dentin bond interface of composite restorations. The null hypothesis that the use of the fluoride mouthwash containing nanohydroxyapatite would not influence the dentin interface microhardness of direct resin composite resin restorations was rejected. This occurred since the final microhardness values were statistically different between the fluoride mouthwash containing nanohydroxyapatite and the placebo fluoride mouthwash containing no nanohydroxyapatite, regardless of the adhesive system tested. The mineralizing agents had been tested to reduce the failure areas of the adhesive interface, hindering the infiltration of harmful agents. Calcium and fluoride have been described in the literature as being able to fill the microporosity present in the hybrid layer, decreasing its permeability [7-10]. As observed in this study, the fluoride mouthwash containing nanohydroxyapatite managed to influence the dentin microhardness showing a possible ability to fill the microporosity present in the dentin interface. However, due to the similar mean values of the initial and final microhardness, it is presumed that despite the capability of the fluoride to penetrate the hybrid layer, it could have been lixiviated from the hybrid layer during the in situ study, not contributing to filling the microporosities present in the dentin interface. The microporosities in the dentin interface occurs due to the incorrect or incomplete penetration of the adhesive monomers into the demineralized dentin, resulting in failure areas within the hybrid layer. These failures within the hybrid layer increase the risk of micro or nanoleakage through the pores present in the adhesive interface, reducing the longevity of the restoration [5-6]. However, the same way oral fluids can infiltrate through these failures, other substances such as calcium and fluoride, present in the saliva or solutions used as mouthwashes [4] could also penetrate and fill the microporosity present in the hybrid layer, thus decreasing its permeability [8-10], and possibly increasing the longevity of composite restorations. As observed in this study, despite fluoride having been described in the literature as being able to fill the microporosity present in the hybrid layer [7-10], the conventional fluoride mouthwash solution (placebo) was not able to increase the initial microhardness much as the fluoride mouthwash containing nanohydroxiapatite. As a limitation of this study, the effects of the fluoride mouthwashes containing nanohydroxyapatite or not were evaluated in situ for only one week. Thus, further clinical studies should be performed to further evaluate the clinical performance of these different treatments using mouthwashes and their real effects in the long term. By all means, the addition of nanohydroxyapatite seems to increase the efficiency of fluoride with regard to mineralization. The remineralizing formula containing nanostructured calcium phosphate, organized in the crystalline form of hydroxyapatite allows the releasing of calcium and phosphate ions to the demineralized surface of the tooth, which can be reorganized in the form of hydroxyapatite, fluorapatite or calcium fluoride, as well as to occlude the dentinal tubules. Moreover, the hydroxyapatite crystals are highly stable and more resistant to acidic challenges when compared to amorphous calcium phosphates, explaining the increased efficiency of the tested formula containing nanohydroxyapatite in comparison to the fluoride placebo formula. Thus, with the limitations of this crossover in situ study, it was possible to conclude that the fluoride mouthwash containing nanohydroxyapatite managed to increase the dentin microhardness of direct resin composite restorations regardless of the adhesive system used within one week. On the other hand, the conventional fluoride mouthwash solution had no influence on dentin microhardness within one week.

\section{Authors contribution}

DO/LM: Experimental design, crossover in situ study coordinator, statistical analysis, manuscript writing, manuscript proofreading. LTP: Experimental design, blinded in situ study clinical instructor, manuscript proofreading. EK: Experimental design, blinded technical microhardness tester, manuscript proofreading. GP/LMP: Experimental design, in situ study clinical monitor, manuscript proofreading.

\section{Acknowledgments}

This study thanks FGM for preparing and donating the mouthwashes used in this study. DO is a Post-Doctoral Researcher at the Sao Paulo Research Foundation (FAPESP grant \#2016/05823-3 and \#2017/22161-7).

The authors declare no potential conflict of interest concerning the authorship or the publication of this article.

\section{References}

1. Buonocore MG. A simple method of increasing the adhesion of acrylic filling materials to enamel surfaces. J Dent Res. 1955;34(6):834-853.

[CrossRef] [PubMed] Google Scholar Scopus

2. Perdigão J. New Concepts in dental adhesion. Northwest Dent. 2000;79(4):29-33.

[PubMed] Google Scholar Scopus

3. Nakabayashi N, Kojima K, Masuhara E. The promotion of adhesion by the infiltration of monomers into tooth substrates. J Biomed Mater Res. 1982;16:265-273. [CrossRef] [PubMed] Google Scholar Scopus

4. Nakabayashi N. The hibrid layer: A resin-dentin composite. Proc Finn Dent Soc. 1992;8(1): 322-329. [PubMed] Google Scholar 
5. Frankenberger R, Perdigão J, Rosa BT, Lopes M. No-bottle vs multi-bottle dentin adhesives - a Microtensile bond strength and morphological study. Dent Mater. 2011;17:373-380. [Full text links] [PubMed] Google Scholar

6. Lopes GC, Marson FC, Vieira LC, et al. Composite bond strength to enamel with self-etching primers. Oper Dent. 2004;29(4):424-9.

[PubMed] Google Scholar Scopus

7. Lenz H. Ultrastructure of the tooth in respect of mineralization, demineralization and remineralization. Int Dent J. 1967;17(4): 693-708.

8. Akimoto N, Yokovama G, Ohmori K, et al. Remineralization across the resin-dentin interface: in vivo evaluation with nanoindentation measurements, EDS, and SEM. Quintessence Int. 2001;32(7):561-570.

[PubMed] Google Scholar Scopus

9. Perdigão J, Van Meerbeek B, Lopes MM, Ambrose WW. The effect of re-wetting agent on dentin bonding. Dent Mater. 1999:15(4):282-95

[Full text links] [PubMed] Google Scholar Scopus

10. Watanabe I, Nakabayashi N, Pashley DH. Bonding to ground dentin by a phenyl-P self-etching primer. J Dent Res.

\section{4;73(6):1212-1220.}

[CrossRef] [PubMed] Google Scholar Scopus

11. Ajami AA, Bahari M, Oskoee SS, et al. Effect of three different mouthrinses o microleakage of composite resin restoration with two adhesive systems after bleaching with $10 \%$ carbamide peroxide. J Contemp Dent Pract. 2012;1(13):16-22. Google Scholar

12. Wilde MG, Delfino CS, Sassi JF, et al. Influence of $0.05 \%$ sodium fluoride solutions on microhardness of resinmodified glass ionomer cements. J Mater Sci Med. 2006;17(9):869-873.

[CrossRef] [PubMed] Google Scholar Scopus

13. Yap AU, Tan BW, Tay LC, et al. Effect of mouthrinses on microhardness and wear of composite and compomer restoratives. Oper Dent. 2003;28(6):740;746.

[PubMed] Google Scholar Scopus

14. Gurdal P, Akdeniz BG, Hakan Sen B. The effects of mouthrinses on microhardness and colour stability of aesthetic restorative materials. J Oral Rehabil. 2002;29(9):895901.

[Full text links] [PubMed] Google Scholar Scopus

\section{Dayane C. R. S. de OLIVEIRA \\ DDS, MS, PhD, Assistant Professor Department of Restorative Dentistry Piracicaba Dental School State University of Campinas Piracicaba, SP, Brazil}

Dayane C. R. S. de Oliveira, DDS, MS, PhD, is a post-doc fellow of the Department of Restorative Dentistry at Piracicaba Dental School, State University of Campinas (UNICAMP), in Brazil. Dr. Oliveira is a young researcher that contributed to 6 textbook chapters, authored 7 patents and is the recipient of many awards in her area of expertise. Her areas of interest include esthetic dentistry, color science and biomaterials development and characterization.

\section{0uestions}

\section{Based on the results in this study, fluoride mouthwashes:}

$\square$ a. Are capable of increasing microhardness of dentin interface of composite restorations; $\square$ b. Are not capable of increasing microhardness of dentin interface of composite restorations; ac. Are not necessarily capable of increasing microhardness of dentin interface of composite restorations depending on composition;

$\square$ d. None of the above.

\section{Based on the results in this study, fluoride mouthwashes containing nanohydroxyapatite:}

$\square$ a. Are capable of increasing microhardness of dentin interface of composite restorations;

b. Are not capable of increasing microhardness of dentin interface of composite restorations;

c. Have similar performance compared to conventional fluoride mouthwashes;

$\square$ d. None of the above.

\section{Based on the discussion section, which statement is true:}

$\square$ a. Fluoride mouthwashes not containing nanohydroxyapatite might be capable of infiltrating in the hybrid layer and forming amorphous calcium phosphates; but it is probably dissolved and lixiviated over time; $\square$ b. Fluoride mouthwashes not containing nanohydroxyapatite do not contribute to filling the microporosities present in the dentin interface over time;

ac. Fluoride mouthwashes containing nanohydroxyapatite are capable of infiltrating in the hybrid layer, and forming highly stable hydroxyapatite crystals;

$\square$. All of the above.

\section{Based on the discussion section, which statement is not true:}

$\square$ a. The primary limitation of this study was follow-up time which does not allow the authors to extrapolate the results in regard the longevity of the composite restorations;

$\square$ b. Literature demonstrate the immediate results of fluoride agents, however, this study demonstrated that these results can be modified over time;

ac. This study demonstrated that increasing the longevity of the composite restorations might be possible using fluoride agents associated with nanohydroxyapatite, however further studies are necessary;

$\square$ d. None of the above. 